\title{
The first wide ultracool binary dwarf in the field: DENIS-J055146.0-443412.2 (M8.5 + L0) ${ }^{\star}$
}

\author{
M. Billères ${ }^{1}$, X. Delfosse ${ }^{2}$, J.-L Beuzit ${ }^{2}$, T. Forveille ${ }^{3,2}$, L. Marchal ${ }^{2}$, and E. L. Martín ${ }^{4}$ \\ 1 European Southern Observatory, Casilla 19001, Santiago 19, Chile \\ e-mail: mbillere@eso.org \\ ${ }^{2}$ Laboratoire d'Astrophysique de Grenoble, Observatoire de Grenoble, BP 53, 38041 Grenoble, France \\ 3 Canada-France-Hawaii Telescope Corporation, 65-1238 Mamaloha Highway, Kamuela, HI 96743, USA \\ 4 Instituto de Astrofísica de Canarias, 38200 La Laguna, Spain
}

Received 18 July 2005 / Accepted 29 July 2005

\begin{abstract}
We present observations of a new very low mass field binary, discovered during an infrared imaging survey of 250 DENIS L and very late-M dwarfs. DENIS-J055146.0-443412.2 is an M8.5 + L0 pair, with a physical separation of over 200 AU. This makes it the widest very low mass binary known in the field, by an order of magnitude. Such a system is fragile, and it would not have survived a close encounter with a third body. Its existence demonstrate that some very low mass stars/brown dwarfs form without ejection from a multiple system, or any other strong dynamical interaction.
\end{abstract}

Key words. stars - brown dwarfs - binary system - formation

\section{Introduction}

Stellar multiplicity is a key observational diagnostic of the star formation process, and of the early dynamical evolution of stellar systems. Different formation scenarios produce distinct multiple star fractions, different distribution functions for separations and mass ratios, and distinct variations of these quantities with primary mass.

The multiplicity of ultracool dwarfs (late-M, L and $\mathrm{T}$ dwarfs) has recently been of particular interest, in part because the leading scenario for their formation makes a very specific prediction. In this "embryo-ejection" scenario (Reipurth \& Clark 2001), dynamical interaction in small sub-clusters eject stellar embryo from their gas reservoir before they can accrete enough mass to become more massive stars. The only binary brown dwarfs expected in this scenario are close binaries that have enough binding energy to survive the $N$-body interactions, and in SPH simulations (e.g. Bate et al. 2003) the few BD binaries produced do have separations below $10 \mathrm{AU}$.

High resolution imaging surveys (Martín et al. 1999; Close et al. 2003; Bouy et al. 2003; Gizis et al. 2003; Burgasser et al. 2003; Siegler et al. 2005) have found a few dozen field ultracool binaries, and indicate that $\sim 10-15 \%$ of the ultracool field dwarfs have a visual companion beyond $\sim 1$ AU. None of these binaries is wider than $20 \mathrm{AU}$, however, in sharp contrast with observed separations above $1000 \mathrm{AU}$ for some systems of early/mid-M dwarfs (Marchal et al. 2003, and in

^ Based on observations collected at the European Southern Observatory, La Silla, Chile. preparation). Imaging of brown dwarfs in young clusters (Neuhäuser et al. 2002; Martín et al. 2003; Luhman et al. 2005; Lucas et al. 2005) demonstrate that wide binaries are already rare at this early stage. The lack of wide very low mass binaries has generally been considered a vindication of the ejection scenario, but the observational statistics are still limited and they do not exclude a low-level tail of wide binaries.

Very recently, Luhman (2004) indeed reported the discovery of a 240 AU binary brown dwarf in the Chameleon I star forming region, and Chauvin et al. $(2004,2005)$ described a planetary mass object orbiting 55 AU from a young brown dwarf in the TW Hydrae Association. The two systems are dynamically fragile, and the strong interactions during ejection from a small sub-cluster would have disrupted them very easily. Both are thus strong evidence against all brown dwarfs forming through ejection. Some skepticism has occasionally been expressed on the physical association of the two Luhman brown dwarfs (e.g. Burgasser et al. 2005), but in our view the case is fairly solid. Both systems were on the other hand found somewhat serendipitously, making the fraction of brown dwarfs with wide companions uncertain. Better ascertaining this small fraction needs homogenous observations of a large sample of ultracool dwarfs.

We are conducting such an observing program for $\sim 250$ dwarfs, and present here its first result, the discovery of the third wide ultracool dwarf binary, and the first in the field. Section 2 briefly presents our overall imaging program, which will be discussed more fully in a later publication, as well as its data for DENIS-J055146.0-443412.2 (hereafter 
Table 1. Photometry and position of the DENIS-J055146.0-443412 system. $I, J, H$ and $K$ are for the combined binary system from the DENIS and 2MASS catalogs; $\Delta J, \Delta K$ and the relative positions were obtained from the SOFI images using the point source mode of the MISTRAL myopic deconvolution package (Mugnier et al. 2004).

\begin{tabular}{lllllll}
\hline \hline \multicolumn{7}{c}{ Astrometry and photometry for the combined DENIS-J055-44 binary } \\
$\alpha(\mathrm{J} 2000)$ & $\delta(\mathrm{J} 2000)$ & $I_{\text {DENIS }}$ & $J_{\text {DENIS }}$ & $J_{2 \text { MASS }}$ & $H_{2 \text { MASS }}$ & $K_{2 \text { MASS }}$ \\
$05: 51: 46.0$ & $-44: 34: 12.6$ & $18.4 \pm 0.3$ & $15.5 \pm 0.2$ & $15.8 \pm 0.1$ & $15.2 \pm 0.1$ & $14.9 \pm 0.1$ \\
\hline \multicolumn{7}{c}{ NTT measurements: observational elements of the binary } \\
& $\rho\left({ }^{\prime \prime}\right)$ & $\theta(\mathrm{deg})$ & $\Delta J$ & $\Delta K$ \\
& $2.20 \pm 0.05$ & 358.6 & $0.55 \pm 0.02$ & $0.53 \pm 0.02$ & \\
\hline
\end{tabular}

DENIS-J055-44). Section 3 presents our infrared spectroscopy of the new binary, and Sect. 4 discusses the implications of its discovery for our understanding of stellar formation.

\section{NTT imaging search for wide companions of ultracool dwarfs}

The imaging sample is drawn from late-M and L dwarfs identified by the DENIS survey, which demonstrated its effectiveness at selecting such objects with the discovery of the first field brown dwarfs (Delfosse et al. 1997). Delfosse et al. (2003) assembled a sample of 300 objects with $I-J>3.0$ (M8 and cooler), from 5700 squares degrees of DENIS survey data. To date we have obtained moderately deep infrared images in the $J$ and $K$ bands for 250 of those 300 DENIS ultracool dwarfs with SOFI, the infrared spectro-imager at the NTT (3.5-m telescope, la Silla, ESO). Our goal is to identify faint companions over the full accessible separation range, from the $0.5-0.8^{\prime \prime}$ typical infrared seeing at La Silla to the $\sim 5^{\prime}$ field of view of the images. We therefore obtain multi-epoch observations to separate true companion from background stars through common proper motion. The companion of DENIS-J055-44 however is sufficiently bright that the DENIS images could provide the first epoch. We therefore marked the pair for early follow-up.

We use SOFI in its Large Field mode, which provides 0 '.288/pixel and a $4.9^{\prime} \times 4.9^{\prime}$ field of view. The observation sequence consists of 5 individual $1 \mathrm{mn}$ exposures acquired on 5 offset pattern within a few arcsecond jitter box. The reduction uses the ECLIPSE package (Devillard et al. 1997). The individual raw data are flat-fielded using a normalized gain map, derived from images of the illuminated dome. The sky signal is estimated from a median across the jittered images and subtracted from the flat-fielded image. Finally, the 5 flat-fielded and sky-subtracted images of each source are shifted and averaged to produce the final image. With nominal weather conditions the $5 \sigma$ limiting magnitude is $J=21.5$.

The NTT images (Fig. 1) show a well separated double star with a moderate contrast of $0.5 \mathrm{mag}$ and a separation of $\sim 2.2^{\prime \prime}$ (Table 1). The shallower and lower resolution DENIS images show a single source that is marginally elongated at $J$ and only barely detected at $I$, with joint magnitudes of $J=15.3 \pm 0.15$ and $I=18.4 \pm 0.3$. DENIS did not detect the target at $K_{\mathrm{s}}$. The lack of any bright source within $15^{\prime \prime}$ of DENIS-J055-44 in the DENIS I image demonstrates that the second component is not a blue background star: even assigning the whole $I$ flux to the fainter component would still leave it with the colour of at most a mid-M dwarf. At the DENIS magnitude limit the sky

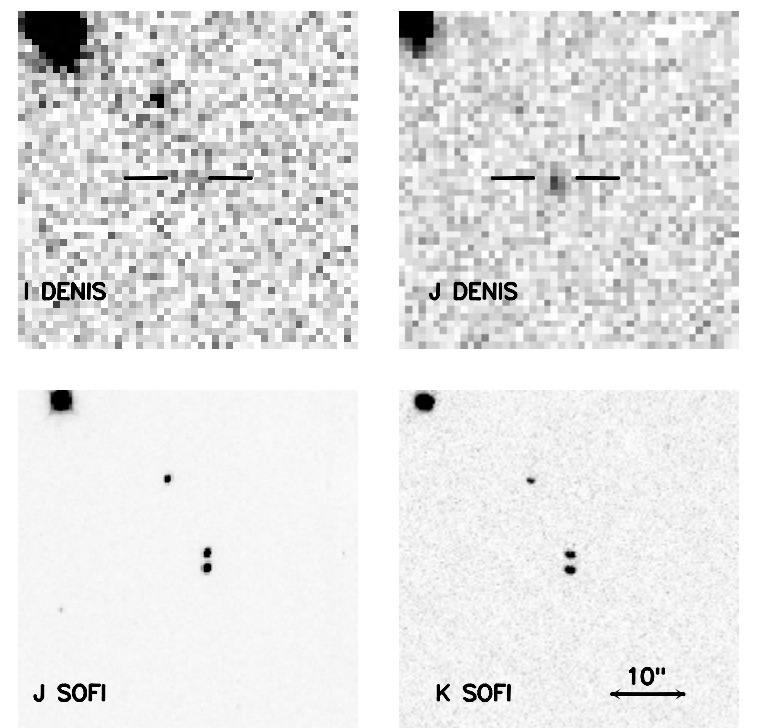

Fig. 1. DENIS discovery images in $I$ and $J$ (top), and SOFI images demonstrating the binary status in $J$ and $K$ (bottom). North is up and East is left.

density of mid-M dwarfs is below 1 per square degree, and the probability to find one by chance within $2.2^{\prime \prime}$ of one of only 250 targets is thus negligible. DENIS-J055-44 also figures in the 2MASS catalog (Cutri et al. 2003) as a single source, with the $J-K$ colour of a late-M dwarf (Table 1).

\section{Infrared spectroscopy}

The spectra were also obtained with SOFI, on the nights of February 9th and 10th 2003. We used the blue and red low-resolution grisms, for dispersions of 6.96 and $10.22 \AA /$ pix, wavelength ranges of respectively $0.95-1.64$ and $1.53-2.52 \mu \mathrm{m}$, and a resolution of 600 with the $1^{\prime \prime}$ slit. SOFI was rotated to align the two components on the slit, and we used the standard observing template for SOFI spectroscopy: the objects are nodded between two positions along the slit, with a small jitter to fill in the bad pixels. The total exposure time is 9 min for the blue part of the spectrum and 6 min for the red part. A standard star (HIP 30374, F5V) was observed immediately after the target for flux calibration.

The spectral images were flat-fielded, sky-subtracted and recombined with a combination of a MIDAS script and ECLIPSE procedures (isaacp package). The images were then rectified, and the spectra of the two components were extracted and wavelength calibrated with IRAF tasks. The wavelength 

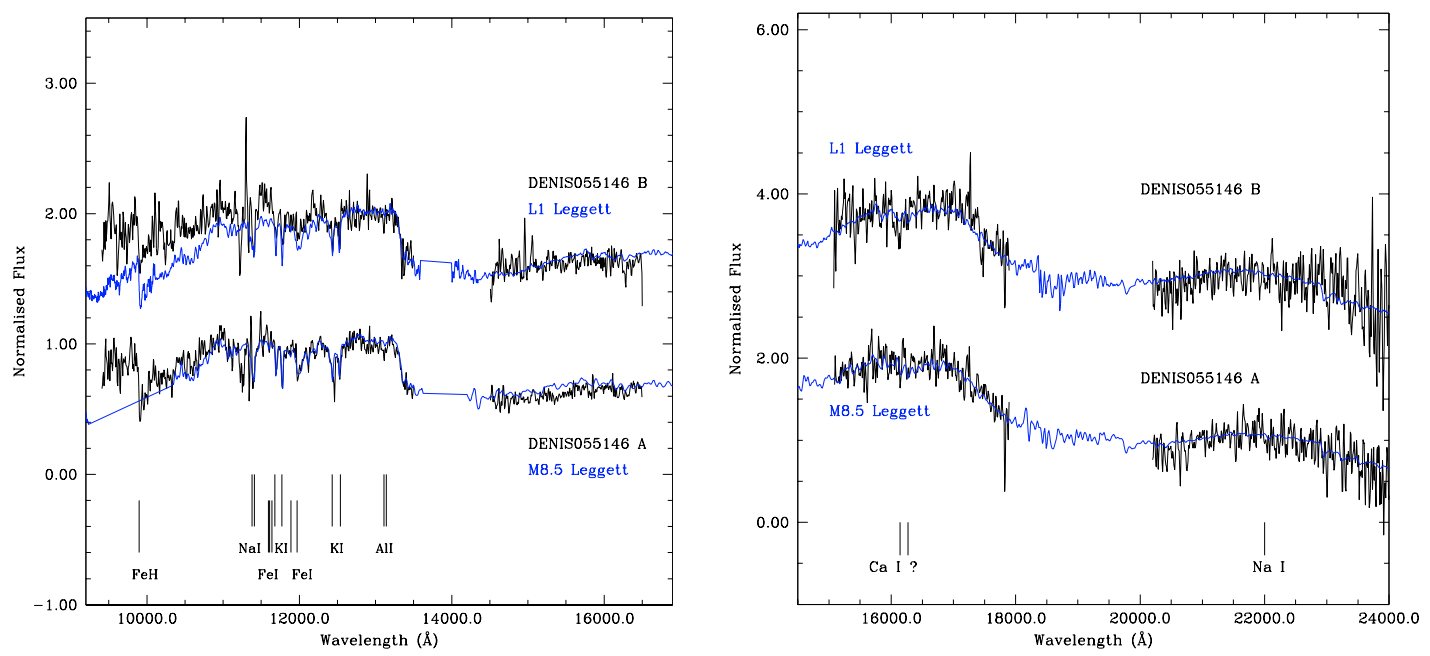

Fig. 2. Blue (left panel) and red (right panel) spectra of the two components of DENIS 0551-44. The blue and red spectra are normalized at respectively $12650 \AA$ and $20800 \AA$, and vertically shifted for clarity. The blue overlays represent spectra of Leggett spectral templates, with spectral types M8.5 for the primary and L1 for the secondary (the Leggett database does not currently contain an infrared L0 spectrum). The integration ranges of the spectral indices are marked at the top, and several characteristic spectral features at the bottom.

calibrations is based on spectra of a Xenon lamp obtained on the same night, and is accurate to better than $1 \AA$. To correct for the instrumental spectral response and for telluric absorption, the spectra were then divided by the ratio of the spectrum of the spectrophotometric standard to a Kurucz model of an F5V star (Kuruzc 1993). Due to the modest altitude of La Silla observatory and the summer observing, the strong telluric water absorption bands are imperfectly corrected. Figure 2 therefore blanks out their wavelength range. The wider stellar $\mathrm{H}_{2} \mathrm{O}$ bands remain conspicuous and demonstrate the late-M/early-L status of the two stars.

The spectra have moderate resolution and relatively poor $\mathrm{S} / \mathrm{N}$ ratios, in particular for the secondary. Our attempts at using spectral indices (Delfosse et al. 1999; McLean et al. 2003) to derive quantitative spectral types were therefore only partly successful. We instead use the full available spectral range, and determine spectral types through least square-matching to the spectral library made available by S. Leggett (http://www.jach.hawaii.edu/pub/ukirt/ $\mathrm{skl} / \mathrm{dM}$.spectra/). In the least square matching we ignore the telluric $\mathrm{H}_{2} \mathrm{O}$ bands $(1.34-1.43 \mu \mathrm{m}$ and $1.80-2.00 \mu \mathrm{m})$, as well as the $\lambda<1.1 \mu \mathrm{m}$ range which is missing for some of the Leggett templates. Both parts of the primary spectrum give a well defined $\chi^{2}$ minimum at spectral type M8.5, while the noisier spectrum of the secondary gives acceptable matches for spectral types M9 to L1. Figure 2 overlays the spectra of the two components with respectively an M8.5 and an L1 template. The M8.5 spectrum clearly matches DENIS 0551-44A very well. The L1 spectrum provides a fair match to DENIS 0551-44B, as does an M9 template, but it leaves some residuals between 1 and $1.2 \mu \mathrm{m}$. This could quite possibly be due to some contamination from light of the close primary, and better quality spectroscopy would be desirable. In the mean time we adopt a spectral type of $\mathrm{L} 0 \pm 1$ for DENIS 0551-44B. The photometric contrast of $\Delta J=\Delta K=$ 0.5 corresponds to a difference of 1.5 spectral sub-type for
late-M or early-L dwarfs (Dahn et al. 2002), and strengthens the adopted classification of the secondary.

The sky density of $I-J \geq 3.0$ (later than M8) objects above the DENIS detection limit $(I=18.5)$ is only 0.052 /square degree (Delfosse et al. 2003). Each component of DENIS 0551-44 being individually fainter than this limit, we conservatively increase the detection volume by $2^{1.5}$. Adopting a constant stellar density (again a slightly conservative assumption), this increases the density of ultracool dwarfs brighter than DENIS 0551-44B to $\lesssim 0.15 /$ square degree. The probability of finding two unrelated late-M or L dwarfs with a projected separation below $5^{\prime \prime}$ is thus $\lesssim 3 \times 10^{-7}$. Moreover, the magnitude difference of the two components is consistent with their spectral type, indicating identical distances to within $\sim 20 \%$. We will soon obtain second-epoch IR images to demonstrate the common proper motion of the pair, but their physical association is already beyond reasonable doubt.

Correcting the 2MASS $J$ magnitude of the system for the companion results in a primary magnitude of $J_{\mathrm{A}}=16.3$. Together with the Dahn et al. (2002) spectral type versus $M_{J}$ relation $\left(M_{J} \sim 11.25\right.$ for M8.5), this results in a spectroscopic distance of $\sim 100 \mathrm{pc}$ for the system and a projected separation between the two components of $\sim 220$ AU.

\section{Discussion}

Numerous previous studies (Close et al. 2003; Burgasser et al. 2003; Bouy et al. 2003; Gizis et al. 2003; Siegler et al. 2005) had found no wide binary ultracool dwarfs in the field, and argued that such systems either never form or must be quickly disrupted during the first few Myr of their life. DENIS 0551-44 demonstrates that wide binary ultracool dwarfs do exist in the field, as they were known to at earlier stages of their evolution (Luhman et al. 2004; Chauvin et al. 2004, 2005). Wide ultracool binaries are undoubtedly rare in the field, and the lack of any detection in previous studies most likely reflects no more than their smaller samples. 
These wide binaries are unexpected in the embryo-ejection scenario, probably the leading theory for the formation of ultracool dwarfs. In current hydrodynamical simulations of the fragmentation of small molecular clouds (Delgado-Donate et al. 2004; Bate et al. 2003), ultracool dwarfs only form in unstable multiple systems, from which they are ejected before they can accrete most of the mass available in their reservoir. These strong early dynamical interactions obviously disrupt any wide binary, because of its weak gravitational bound.

One major motivation behind the embryo-ejection scenario is that the typical thermal Jeans mass in molecular cloud cores is $\sim 1 M_{\odot}$ (Larson 1999), so classical gravitational collapse only forms stars and no brown dwarfs. Padoan \& Nordlund (2004), however, show that accounting for turbulence allows gravitational collapse to form brown dwarfs as well: supersonic turbulence generates highly nonlinear density and velocity field in star-forming clouds, invalidating the quasi-static Jeans gravitational stability criterion. Magnetic fields in addition most likely play an important role in the fragmentation/collapse process and their effects on the IMF are poorly understood. Brown dwarfs (and object at the stellar-substellar boundary) could thus well form like stars, in which case the small number of wide binaries rather reflects a posterior stage of dynamical decay for most weakly bound systems (Sterzik \& Durisen 2003).

An alternative formation process for ultracool dwarfs is the photo-evaporation model (Kroupa \& Bouvier 2003; Whitworth \& Zinnecker 2004), where very low mass objects form in the vicinity of $\mathrm{OB}$ stars whose strong UV fields erodes the outer layers of protostellar cores before reach a stellar mass. This mechanism is only effective in (very) dense star formation regions, and highly wasteful of the available gas. It could thus be responsible for a few very low mass objects, but not for a majority.

DENIS 0551-44 clearly did not form through embryoejection, but could be the result of either fragmentation, like more massive stars, or of photo-evaporation in the vicinity of OB stars. As previously discussed by Luhman (2004), the existence of a few very wide ultracool binaries demonstrates that some brown dwarfs form outside the embryo-ejection scenario, though not that all of them $\mathrm{do}^{1}$. The proper-motion analysis of our full sample will shortly provide a good measurement of the fraction of such wide binaries among ultracool dwarfs. This statistics will constrain the relative contributions of the various modes of brown dwarf formation.

Acknowledgements. We thanks the NTT team for their precious help during the observations which led to these results. We are grateful to the referee, K. L. Luhman for suggestions that improved the paper and a prompt report. Financial support from the "Programme National de Physique Stellaire" (PNPS) of CNRS/INSU, France, is gratefully acknowledged. DENIS is the result of a joint effort involving human and financial contributions of several Institutes mostly located in Europe. It has been supported financially mainly by the French

1 A proviso to this statement is that DENIS 0551-44 could in principle be of higher multiplicity, and hence have a larger mass than is apparent. Higher resolution imaging would be of interest to ascertain this.
INSU, CNRS, and French Education Ministry, the ESO, the State of Baden-Wuerttemberg, and the European Commission under networks of the SCIENCE and Human Capital and Mobility programs, the Landessternwarte, Heidelberg, l'Institut d'Astrophysique de Paris, the Institut für Astrophysik der Universitat Innsbruck and Instituto de Astrofisica de Canarias.

\section{References}

Bate, M. R., Bonnell, I. A., \& Bromm, V. 2003, MNRAS, 339, 577 Bouy, H., Brandner, W., Martín, E. L., et al. 2003, AJ, 126, 1526

Burgasser, A. J., Kirkpatrick, J. D., Reid I. N., et al. 2003, ApJ, 586, 512

Burgasser, A. J., Kirkpatrick, J. D., \& Lowrance, P. J. 2005, AJ, 129, 2849

Chauvin, G., Lagrange, A.-M., Dumas, C., et al. 2004, A\&A, 425, L29

Chauvin, G., Lagrange, A.-M., Dumas, C., et al. 2005, A\&A, in press [arXiv: astro-ph/0504659]

Close, L. M., Siegler, N., Freed, M., \& Biller, B. 2003, ApJ, 587, 407

Cutri, R. M., Skrutskie, M. F., Van Dyk, S., et al. 2003, 2MASS All Sky Catalog of Point Sources; VizieR On-line Data Catalog II/246 Dahn, C. C., Harris, H. C., \& Vrba, F. J. 2002, AJ, 124, 1170

Delfosse, X., Tinney, C. G., Forveille, T., et al. 1997, A\&A, 327, L25 Delfosse, X., Tinney, C., Forveille, T., et al. 1999, A\&AS, 135, 41

Delfosse, X., Forveille, T., Martín, E. L., et al. 2003, SF2A-2003, 585

Delgado-Donate, E. J., Clarke, C. J., Bate, M. R., \& Hodgkin, S. T. 2004, MNRAS, 351, 617

Devillard, N. 1997, The Messenger, 87, 5

Gizis, J. E., Reid, I. N., Knapp, G. R., et al. 2003, AJ, 125, 3302

Kroupa, P., \& Bouvier, J. 2003, MNRAS, 346, 369

Kuruzc, R. L. 1993, ATLAS9 Stellar Atmospheres Programs, Grids of Model Atmosphere and Line Data Smithsonian Astrophysical Observatory, Cambridge, MA

Larson, R. B. 1999, in Star Formation, ed. T. Nakamo, Nobeyama Radio Observatory, 336

Lucas, P. W., Roche, P. F., \& Tamura, M. 2005, MNRAS, in press [arXiv: astro-ph/0504570]

Luhman, K. L. 2004, ApJ, 614, 398

Luhman, K. L., McLeod, K. K., \& Goldenson, N. 2005, ApJ, 623, 1141

Marchal, L., Delfosse, X., Forveille, T., et al. 2003, IAUS, 211, 311

Martín, E. L., Brandner, W., \& Basri, G. 1999, Science, 283, 1718

Martín, E. L., Barrado, y Navascués D., Baraffe I., et al. 2003, ApJ, 594, 525

McLean, I. S., McGovern, M. R., Burgasser, A. J., et al. 2003, ApJ, 596, 561

Mugnier, L. M., Fusco, T., \& Conan, J.-M. 2004, J. Opt. Soc. Am. A., 21(10), 1841

Neuhäuser, R., Brandner, W., Alves, J., Joergens, V., \& Comerón, F. 2002, A\&A, 384, 999

Padoan, P., \& Nordlund, A. 2004, ApJ, 617, 559

Reipurth, B., \& Clark, C. 2001, AJ, 122, 432

Siegler, N., Close, L. M., Cruz, K. L., Martín E. L., \& Reid, I. N. 2005 ApJ, 621, 1023

Sterzik, M. F., \& Durisen, R. H. 2003, A\&A, 400, 1031

Whitworth, A. P., \& Zinnecker, H. 2004, A\&A, 427, 299 